\title{
Identifying Aspects of Ambient Intelligence Through A Review of Recent Developments
}

\author{
Maria Charalampidou, Spyridon Mouroutsos and George Pavlidis \\ Department of Electrical and Computer Engineering, Democritus University of \\ Thrace, Xanthi, GR-67100 Greece \\ E-mail: macharal@ee.duth.gr \\ Department of Electrical and Computer Engineering, Democritus University of \\ Thrace, Xanthi, GR-67100 Greece \\ E-mail: sgmour@ee.duth.gr \\ 'Athena' Research and Innovation Center, Xanthi, GR-67100, Greece \\ E-mail: gpavlid@ceti.gr
}

\begin{abstract}
It has already been realized by the scientific and technical community that a new form of technology is going to lead the future technological developments. This technology will be more humancentric and will be more and more "hidden" within everyday-life objects. It will be smarter, personalized, pervasive and ubiquitous. This technology includes what is called Ambient Intelligence (AmI). In this paper, we identify the main aspects of AmI through a review of the recent developments that have been achieved in these aspects of AmI and Ambient Intelligence Environments (AmIEs), as well as point out the problems yet to be solved and the visions of the future.
\end{abstract}

Keywords: Ambient intelligence, intelligent interfaces, human-machine interaction.

\section{Introduction}

Ambient intelligence (AmI) is the vision of a technology that will become invisibly embedded in our natural surroundings, present whenever we need it, enabled by simple and effortless interactions, attuned to all our senses, adaptive to users and context-sensitive, and autonomous. High-quality information access and personalized content must be available to everybody, anywhere, and at any time. From the technical standpoint, distributed electronic intelligence is addressed as 
hardware vanishing into the background. Devices used for AmI are small, lowpower, low weight, and low-cost; they collaborate or interact with each other; and they are redundant and error-tolerant. This means that the failure of one device will not cause failure of the whole system. Since wired connections often do not exist, radio methods will play an important role for data transfer [1].

Eli Zelkha, Brian Epstein and Simon Birrell are thought of having coined the term "Ambient Intelligence") in 1998 [2]. The term ambient refers to the environment and reflects the need for typical requirements such as distribution, ubiquity, and transparency [3]. It is important to mention here that the difference between Ubiquitous Computing and Ambient Intelligence, is the more technology/networks-centered focus of the former and the more interdisciplinary nature of the latter that considered human and societal factors, although there have been arguments about this distinction [4].

Philips was one of the first to invest on AmI research, since 1998, starting with an investigation on different scenarios that would transform the high-volume consumer electronic industry from the current "fragmented with features" world into a world where user-friendly devices support ubiquitous information, communication and entertainment. Almost concurrently, the MIT Project Oxygen [5] was launched aiming at developing technology that is human-centric. The Oxygen alliance was formed for industrial partners. It was soon realized that there was a need to construct a feasibility and usability facility for AmI and planning started in 2000 that led to the launching of Philips HomeLab [6] in 2002. A number of other initiatives started to explore AmI along with Philips. The European Commission's Information Society Technology Advisory Group (ISTAG) with a number of documents and recommendations [7]-[9], led to the inclusion of AmI in the sixth framework (FP6) in IST. In addition, MIT started an AmI research group at Media Lab and the Fraunhofer Society initiated activities in various AmI domains. 2004 was the year that the first European symposium on AmI (EUSAI) took place while other conferences have been organized to address specific topics within AmI.

The idea of AmI is not without opponents. In [10] the authors make a "warning" to policy-makers, industry, academia, civil society organizations, the media and the public about the threats and vulnerabilities facing our privacy, identity, trust, security and inclusion in the rapidly approaching world of AmI. Embedded within the very concept of AmI, are technologies as ubiquitous computing, communications and interfaces converge and adaptation to the user, in the promise of user-friendliness, transparent and seamless user recognition and responding. The immersive, context-aware, personalized, anticipatory characteristics of AmI could bring up various concerns regarding intrusions in personal privacy, individualization of society, concentration of power in large organizations, and intermixing and diffusion of virtual with real. Even in the typical "Ellen returns home after a long day's work" AmI example, there are at least two references to the term "surveillance". As a result there is ongoing 
research regarding the investigation of the socioeconomic, political and cultural aspects of AmI. To deal with the same aspects, the European Commission IST Advisory Group made specific suggestions that would permit the societal acceptance of ambient intelligence. According to these suggestions, AmI should:

- facilitate human contact

- be oriented towards community and cultural enhancement

- help to build knowledge and skills for work, better quality of work, citizenship and consumer choice

- inspire trust and confidence

- be consistent with long term sustainability - personal, societal and environmental — and with life-long learning.

- be made easy to live with and controllable by ordinary people.

In this paper, inspired by the tremendous potential of AmIEs as well as by the current progress in the corresponding fields of research, we identify the individual aspects of AmI through an overview of the current research directions and applications as well as future challenges.

The remainder of this paper is organized as follows: After providing the typical AmI usage example, Section 2 highlights the aspects of AmI that can be identified and the research that has been conducted in each aspect. Section 3 presents the latest applications on AmI and Section 4 discusses the future of AmI.

\subsection{The Typical Example}

"Ellen returns home after a long day's work. At the front door she is recognized by an intelligent surveillance camera, the door alarm is switched off, and the door unlocks and opens. When she enters the hall the house map indicates that her husband Peter is at an art fair in Paris, and that her daughter Charlotte is in the children's playroom, where she is playing with an interactive screen. The remote children surveillance service is notified that she is at home, and subsequently the on-line connection is switched off. When she enters the kitchen the family memo frame lights up to indicate that there are new messages. The shopping list that has been composed needs confirmation before it is sent to the supermarket for delivery. There is also a message notifying that the home information system has found new information on the semantic Web about economic holiday cottages with sea sight in Spain. She briefly connects to the playroom to say hello to Charlotte, and her video picture automatically appears on the flat screen that is currently used by Charlotte. Next, she connects to Peter at the art fair in Paris. He shows her through his contact lens camera some of the sculptures he intends to buy, and she confirms his choice. In the mean time she selects one of the displayed menus that indicate what can be prepared with the food that is currently 
available from the pantry and the refrigerator. Next, she switches to the video on demand channel to watch the latest news program. Through the follow me she switches over to the flat screen in the bedroom where she is going to have her personalized workout session. Later that evening, after Peter has returned home, they are chatting with a friend in the living room with their personalized ambient lighting switched on. They watch the virtual presenter that informs them about the programs and the information that have been recorded by the home storage server earlier that day."

\section{The aspects of AmI}

In order to consider an environment as AmIE, it is essential to give a definition to the terms environment and AmIE. Environment refers to a system consisting of humans or other living beings, such as animals or plants, objects, varying from a common pen to the most complex gadget, activities, varying from sleeping to bungee jumping or health care needs. Such an environment can be considered as AmIE as long as it can accomplish the following tasks:

- Listen

- Respond

Listen means that the environment can always recognize and comprehend the physical entities of the environment. To accomplish this aspect, modeling the physical entities is crucial for the system. Modeling the environment, allows the digital representation and the continuous interpretation of the information regarding the system. In practice, this aspect includes not only recording information from multiple sensor inputs but also evaluation, which is made through the classification of the information. Respond means that the environment can serve the requirements of the environment. To accomplish this aspect the system must process the information in order to define the specific 'needs' of the environment and plan decisions and actions to serve them. This aspect, in practice, besides the action upon the environment also includes interaction with humans. A combination of these two main aspects enables the system to learn continuously about the special requirements and actions that need to be taken. And this is the aspect of intelligence.

After reviewing the recent developments in AmI and AmIE, we have concluded that the following aspects of AmI can be identified and distinguished, under the general Listen-Respond operation:

- Listen

- Modeling the environment

- Gathering information of multiple sensor inputs

- Classification of the information 
- Respond

- Processing of the information

- Planning decisions and actions

- Interaction with humans

- The aspect of intelligence

\subsection{Modeling the environment}

Modeling the environment is the first and an inextricable step to create an AmIE. To accomplish modeling of the environment researchers use agents as a good way to model, simulate, and represent meaningful entities such as rooms, cars, or even persons [12].

A multiagent architecture is described in [13], where the authors describe how their architecture, consisting of distributed embedded agents, utilizes sensory information to learn to perform tasks related to user comfort, energy conservation, safety and monitoring. They show how these agents, employing a behavior-based approach (derived from robotics research), are able to continuously learn and adapt to individuals within a building, whilst always providing a fast, safe response to any situation.

Ontology has been introduced into the AmI domain as a means to provide declarative formal representations of the domain knowledge. In [14], the authors present a prototype ontology-based representation for Universal Plug-n-Play (UPnP) devices and services that provide a semantic linking between humancentric abstract descriptions and the software-centric concrete description that derives from the UPnP Extensible Markup Language based (XML) descriptors. The resulting ontology has rich semantics that can be combined with other higher level domain ontologies and used by ontology-based systems.

A novel multiagent system based on distributed coordination reasoning under uncertainty for sustain-ability called SAVES (Sustainable multi-Agent systems for optimizing Variable objectives including Energy and Satisfaction) is given in [15]. The authors argue that there are three key ideas in SAVES:

- it explicitly considers uncertainty while reasoning about coordination in a distributed manner relying on Modeling Multiagent Coordination (MDP)

- human behaviors and their occupancy preferences are incorporated into planning and modeled as part of the system

- influence of various control strategies for multi-agent teams is evaluated on an existing university building as the practical research test-bed with actual energy consumption data 
They also, empirically show the preliminary results that their intelligent control strategies substantially reduce the overall energy consumption in the actual simulation test-bed compared to the existing control means while achieving comparable average satisfaction level of occupants.

\subsection{Gathering information of multiple sensor inputs}

The inputs of an AmI system are signals from sensors that can be delivered either through wires or wireless. The task of gathering information from these multiple sensor inputs includes analyzing various sensing inputs including speech recognition and machine vision [12].

Despite the fact that IEEE 802.15.4 networks, along with other wireless protocols, such as Zigbee and Bluetooth, are promising solutions for wireless personal area networks, and in particular for wireless home area networks, real-world trials with 802.15.4 devices are difficult, because of the characteristics of these devices (small dimensions, wireless communication), and the potentially large size of the network. The authors in [16], present an IEEE 802.15.4 network emulation testbed that can be used to perform experiments with 802.15.4 applications in a controlled virtual environment.

Various gesture recognition techniques for inter-facing machines based on AmI have been reported in [17], where the approaches for gesture recognition are divided into two categories: data-glove based and vision based. Moreover eye detection methods are divided into two major categories: traditional image-based passive approaches and active IR-based approaches.

In [18] the authors discuss an approach that uses natural language processing and information extraction toolkits to identify entities and statements about their spatial relations. These extractions are then processed by a spatial reasoner to convert them from the human conceptual space into the quantitative space used by the computer-based agent.

A survey has been made regarding the field of visual sensor networks in [19], where the authors provide an overview of the current state-of-the-art in the field of visual sensor networks, by exploring several relevant research directions. Visual sensor networks (VSNs) consist of tiny visual sensor nodes called camera nodes, which integrate the image sensor, embedded processor, and wireless transceiver [19].

\subsection{Classification of the inputs}

While AmI represents a vision of an environment where both technology and human activities enjoy a combination of ultimate harmony, this could not be achieved without a certain classification of the inputs. This task is being considered under the scope of the context. To make clear the significant role that con-text information plays to this vision, one has just to mention that without 
context information AmI would be a simple "if... then" model. Context-aware computation means that the system automatically senses the context; the context changes the historical context, and then adjusts its behavior in accordance with its own judgment. In generally, context can be divided into [20]:

- Environmental context, such as time, temperature and other environmental factors

- Appliance context, such as the state of an appliance and

- User context, such as user's identity, the habits, the interests, and so on

Because of the fact that there are various and many contexts in AmIEs, contexts are needed to be classified according to characteristics and purposes [21]. For instance, context classification in [21], includes linguistics, psychology and computer science.

Context modeling is an issue that has been extensively researched. An approach to context modeling is given in [22], where a generic context model, which consists of three fundamental classes, namely Extrinsic Context, Interface Context, and Intrinsic Context is proposed. The goal of the proposed framework is to represent information context, in general, which can facilitate common context representation, context matching, and context reasoning.

A recent study about context is given in [23], where the authors describe the SimCon (Simulated Context) Generator, which combines data on the state of a Virtual Reality building with the SimCon Model to generate interactive location context for the rapid evaluation of Smart Building Applications. The paper evaluates the simulated context against physical readings.

\subsection{Processing of the information}

In general, information is processing with Bayesian networks, fuzzy logic, and rough sets [12]. In [24] the authors present a survey of the basic theory of some of the most common methods of natural computing applied to the context of mechanical systems research. Specifically, this work includes: data and sensor fusion, feature extraction and selection, artificial neural networks, fuzzy sets and fuzzy logic, statistical learning theory and kernel methods, as well as natural optimization methods. The paper also attempts to give some indication as to which of the algorithms emerging now from the machine learning community are likely to be important for mechanical systems research in the future.

In another study [25], the authors propose a model for situation modeling and identifying under uncertainty. The semantic of a situation may be fuzzy; context usually comes with imperfections such as imprecision and incompleteness. In this model, fuzzy ontology based on the authors' new fuzzy language, F-OWL, is used to model context and situation, which can make the structure and semantic of context and situation easily understood, reused and shared by users, devices and 
services. Moreover, a situation approximate identifying algorithm is proposed to identify situations considering uncertainty.

\subsection{Planning decisions and actions}

This task includes planning for problem solving by producing a sequence of action needed to achieve a particular goal. A type of decision planning is presented in [13], where the authors expand the meaning of a Smart Home to a Semantic Smart Home. Semantic Smart Homes contain an additional semantic layer that aims to reach goals such as energy efficiency and optimization, provision of comfortable indoor environment, inhabitants' security and safety management. This may be performed by running competitive software agents.

Another study on planning decisions is given in [26], where the Sentient project is discussed, which intends to detect negative emotional reactions using physiological signals, inform the user, and orchestrate a user-defined response from the environment to provide emotion regulation. They aim at coordinating a system response that may ameliorate the negative consequences of emotional reactions, i.e. AmI mediated self-regulation. In particular, the authors present the course of action taken for initial user involvement in their design where User Centered Design techniques are used in order to build a realistic system that reflects and satisfies existing user needs.

Moreover, research has been made on actions depending on partial knowledge. In [27] it is demonstrated how a recently proposed epistemic action theory from the field of cognitive robotics can contribute in facilitating different aspects of AmI. In this paper the authors describe different aspects of research in AmI, to which reasoning under partial knowledge can contribute, providing also examples from an implemented system that couples rule and causality-based techniques. A formal theory for reasoning about knowledge, actions and causality, the Discrete Event Calculus Knowledge Theory (DECKT) [28] is applied. This theory assumes agents acting in dynamic environments, having accurate but potentially incomplete knowledge and able to perform knowledge-producing actions and actions with context-dependent effects.

\subsection{Interacting with humans}

AmI systems should be able to interact intelligently with humans. Such interaction requires context awareness. Here lies one of the main goals of AmI, which is to provide with an interaction interface that is transparent to the user. Since emphasis is given to the user (user-centric design) AmI should provide interfaces according to everyday real-life objects and human habits, while hiding itself and requiring no technical training or knowledge from the user. An example in this field is given in [29], where the authors describe a rather unconventional approach both to AmI and education. They argue that the interfaces used, should be simple and make sense to the children using computers that should be hidden and the 
interaction should take place through familiar play objects to which the children already have a connection.

Moreover according in [30] the authors have developed novel robust methods for human action recognition. [31] introduces a novel, real-time approach for smile detection. Also, [32] introduces a system that facilitates rapid development of interactive systems and [33] presents many examples of work on control of virtual characters in theatrical performances. Increasing research and development during recent years has explored Human-Robot interactions especially for the challenge of service robots. In [34] the authors explore the use of human-robot interaction applied to telepresence. More specific, two service robots are presented, Walkabot and Snowie. Snowie is currently used for emotion expression in human-robot interactions, whereas Walkabot is used to help the elderly and rehabilitation.

In [35], data collected at a training session for health care personnel and alarm operators in steering a mobile social telepresence robot for the first time is presented. The purpose of the system is to be used as a communicative tool particularly when interacting with an elderly audience. Additionally, in this study it was investigated how intuitive the system usage was as well as how attentive the users were to what was going on in the environment. The overall results presented in the paper, are based on questionnaires, which include questions about experienced social and spatial presence and suggest that they are indeed suitable for use also in the social robotic telepresence domain for providing indications on both social and spatial presence.

\subsection{The aspect of intelligence}

A combination of the two basic aspects of AmIE, Listen and Respond, can be found in [36]. In this paper the researchers present "ePH", an acronym for "ePrahova", where Prahova is the name of their county, for which the system has been developed. The a system provides sharing of public interest information and knowledge that can be accessed via always-on, context-aware services, focusing on its multi-agent architecture and on its multi-dimensional context model. A multi-agent architecture of a con-text-aware system, which is called "FACE", and the learning scenarios within ubiquitous learning environments that the system supports are also presented.

In [37], the authors propose PALTA: Peer-to-peer Adaptable Topology for Ambient intelligence, in order to develop a system more acceptable to humans, enabled by basic machine learning methods that allow it to learn by observing users. A decentralized and self-adaptable network topology is used. According to the authors, PALTA is a self-organized and self-managed network topology, which can optimize the use of the network and device resources depending on the network conditions.

Current activity recognition approaches usually ignore knowledge learned in previous smart environments when training the recognition algorithms for a new 
smart environment. In [38], it is proposed a method of transferring the knowledge of learned activities in multiple physical spaces, e.g. homes $\mathrm{A}$ and $\mathrm{B}$, to a new target space, e.g. home C. This method introduces a new way of making an environment intelligent by transferring the knowledge of learned activities to a target space. Furthermore the method results in reducing the data collection and annotation period, achieving an accelerated learning pace and exploiting the insights from previous settings.

\section{Applications of AmI}

Examples of AmI applications can be found in several environments such as smart homes, smart offices, intelligent meeting rooms, ambient healthcare, smart classrooms [21].

However, due to a lack of smart home infrastructures for experimental purposes, it is difficult for researchers to conduct satisfactory experiments in concrete environments with real data. An effort to address this issue has been made with SIMACT (SIMulator for ACTivity recognition). As presented in [14], SIMACT is a new 3D smart home infrastructure simulator (Fig. 1) developed in Java specifically to help researchers working in the field of activity recognition. A set of prerecorded scenarios, made with data gathered during clinical trials, are included with the simulator in order to provide ground truth data for testing activity recognition algorithms. The goal, as mentioned in the paper, was to release the SIMACT simulator as an open source component that would benefit the whole smart home research community.

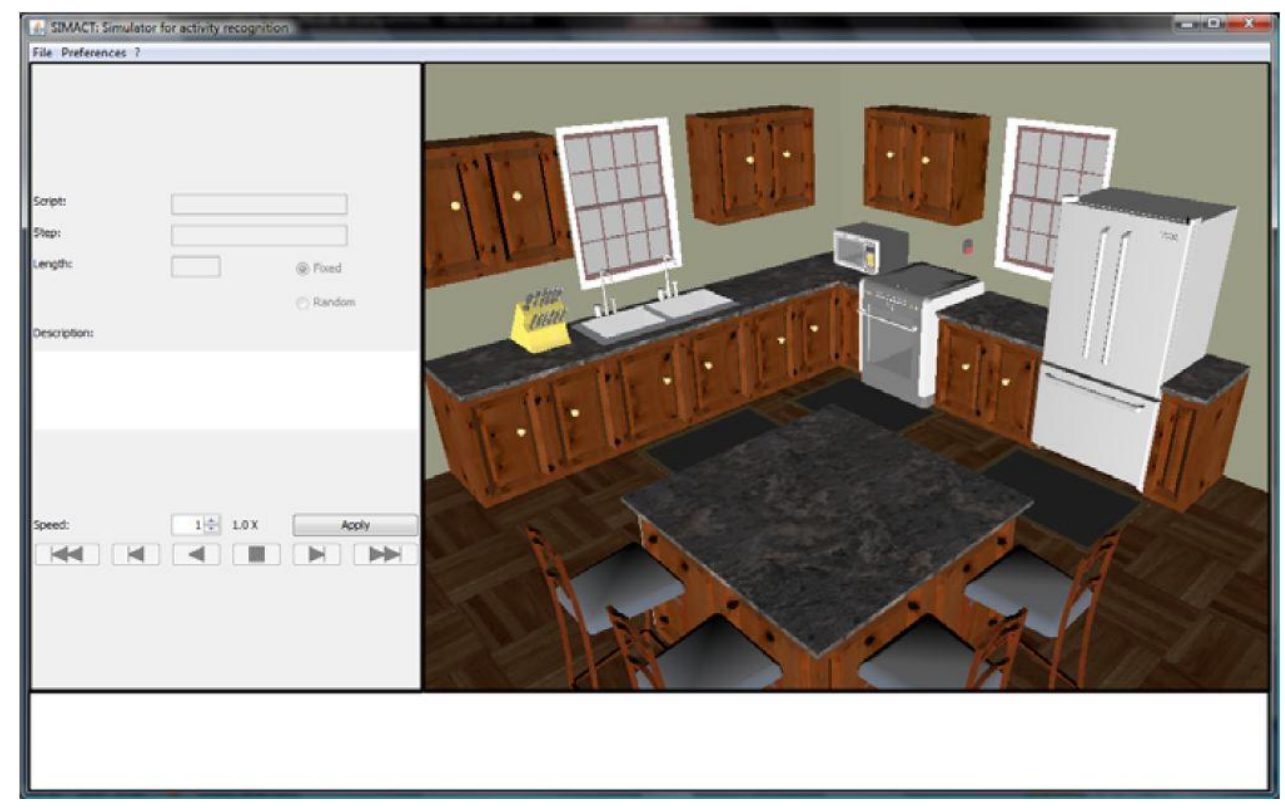

Fig. 1. SIMACT in action 
In [19], the authors discuss about the holistic realization of practical "AmISpace", a system that has been applied in the AmI and embedded systems that is supported by the 211 project of Beijing University of Technology.

AmI has been applied in wireless home automation networks (WHANs) that comprise wireless embedded sensors and actuators that enable monitoring and control applications for home user comfort and efficient home management. The article in [39] surveys the main current and emerging solutions that are suitable for WHANs, including ZigBee, Z-Wave, INSTEON, Wavenis, and IP-based technology.

AmI has not only been applied in health care and business but in our everyday life too, through ambient lifestyle feedback systems, which include a feedback loop between the users' behavior and the expression shown on an ambient display. Thus applications in an AmIE must influence user's behavior appropriately. In [40], the authors discuss how ambient lifestyle feedback systems based applications affect users in changing their habits using four case studies. Each case study is designed to use different persuasion techniques in order to confirm whether those techniques are effective or not [40].

An RFID-based system was developed in [41] for home safety. Dangerous situations for both elders and children can be avoided through warning issuing to the caregiver. Fuzzy rules and fuzzy inference are used in the system.

AmI can be applied in fields such as urban computing where streaming space/environment information are provided to the users (Fig. 2). Although in such a field there is an issue with security, there have been proposed algorithms that enhance the security between users and the environment like authentication, and ways to secure the content in urban computing as presented in [42]. However, the technology in this field seems yet to be immature.

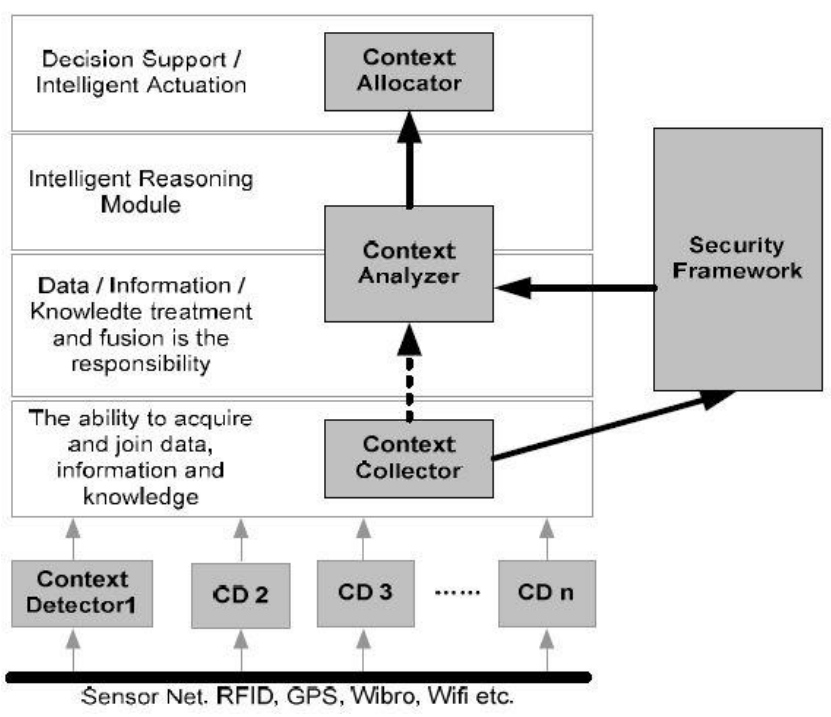

Fig 2. Security functions 
A lot of research has been conducted in the field of developing strategies and schemes for serving disabled people. In [43], for instance, the researchers analyze the possibilities of integrating different technological and knowledge representation techniques for the development of a framework for the remote control of multiple agents' activities, in order to recognize the prediction criteria of diagnoses of the emotional situations of disabled persons, such as wheelchairtype robots.

A novel Camera Canvas, photo editing and picture drawing software for individuals who cannot use their hands to operate a computer mouse has been developed in [44]. Camera Canvas is designed for use with camera-based mousereplacement interfaces that allow a user with severe motion impairments to control the mouse pointer by moving his or her head in front of a web camera. To make Camera Canvas accessible to as wide of a range of movement abilities as possible, the researchers designed its user interface so that it can be extensively tailored to meet individual user needs.

An awareness system that was designed to provide peace of mind and a sense of connection to adults, who care for an elderly parent living alone, is presented in [45]. The authors show the iterative design of the Aurama awareness system (Fig. 3 ) and their empirical research in addition to the role that formal care providers can play when caring for the elderly using the awareness system. They also discuss implications of their studies for the design of ambient intelligent systems supporting awareness between elderly, their adult children and care providers.

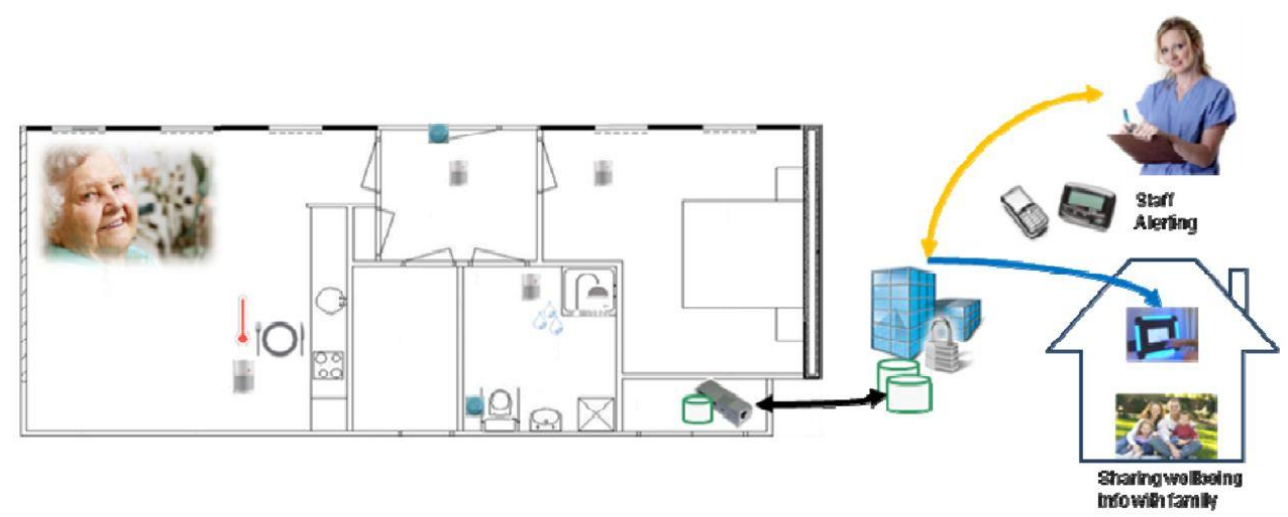

Fig 3. Third generation of the Aurama awareness system: towards less obtrusive sensing and involving formal care providers.

In [46] the authors present a new development method that is based on a detailed three-dimensional model and show how the model can be used for designing and evaluating assistive environments. They present an architectural foundation of how a Universal Remote Console technology can be applied to synchronize the virtual environment model with the real world. Furthermore, the authors extend previous work on Dual Reality by introducing the concept of Synchronized Realities that include the synchronization between remote AmI environments. The 
authors also discuss application scenarios and show their system at work using two demonstrators that they implemented based on the proposed development method.

Aside from the above applications researchers in [47] present a framework in the field of eTourism that is a context-based matchmaking approach, which addresses the needs of tourists during their trip and aims to provide a more satisfying visit experience.

Another study in [48] present an application in order to make museum visits more enjoyable to small groups using drama-based presentations that contain slight differences in content between any two visitors, leveraging the narrative tension/release cycle to naturally lead visitors to fill in missing pieces by interacting with friends and initiate a conversation.

Location-aware ambient environments are relevant for using interactive virtual characters. The authors in [49] designed a location-aware virtual agent that adapts its spatial behavior to users' and objects' locations during a search task in a smart room. In this paper the authors explain how they designed a location-aware virtual agent that helps users find objects in a smart room. According to their results a location-aware adaptive agent elicited higher levels of perceived presence and perceived adaptivity. Furthermore, performance was less influenced by task difficulty when users interacted with the adaptive agent.

\section{Issues open for research in AmI}

AmIEs with numerous of sensors and actuators as well as mobile and workstation devices, need to be environments not only flexible and adaptive but also smart. There are a number of problems yet to be properly addressed to make the development of such systems possible. These problems include mutual understanding between heterogeneous agents, a new architecture specification for general-purposed agents and their social system, as well as an Application Programming Interface for the development of social agent-based systems as referred in [50]. A method for mutual understanding among agents is Knowledge request Broker Architecture (KnoRBA) that is proposed in this paper. Besides, in [51] the authors propose an approach to resolve conflicts between policies of different administrative domains at runtime by means of meta-policies.

As AmI can be consider as an extended hardware and software system, which is continuously affected by changes in its execution environment (infrastructural variation or a modification of the user requirements, or changes in the environment) the combinatorial explosion of the number of potential configurations must be addressed by researchers. Thus, Dynamically Adaptive Systems (DASs) have to be developed by defining several variation points. Depending on the context, the system dynamically chooses suitable variants to realize those variation points. These variants may provide better quality of 
service, offer new services that did not make sense in the previous context, or discard some services that are no longer useful [52]. In [52], the authors propose a framework called F4Plan to ease the integration of different planning algorithms that produce a more efficient adaptation plan than an ad-hoc algorithm.

Indisputably, the applications of AmI promise a more comfortable life but complex legal and social issues arise, in terms of fundamental rights and freedom. The privacy of the users, in contemporary and future AmIE is an issue discussed in a number of papers. A solution to this problem is proposed in [53], where "Activity spheres" (a model to everyday activities that are carried out within such an environment) protect the privacy of each user by applying privacy policy ontology, called PROACT.

\section{The future of AmI}

Aside from the technical and legal issues that must been addressed, there is a promising future ahead for AmI. A view of this future is given in [54], where the authors illustrate their approach with a task analysis of a scenario that shows the challenges of "Next Generation Ambient Intelligence Environments", which first introduced in [55] and connect the earlier architectures that supported objects connecting with each other in order to form a ubiquitous computing system with ambient ecologies consisting of objects always connected to the network. In [54] the authors propose ontology prototypes that are required for the implementation of their scenario. Their approach is based on an agent-based, service-oriented architecture, a combination of heterogeneous ontologies and ontology matching algorithms, which maximizes the use of available interaction resources, while decoupling interaction specification from interfaces and modalities. A new approach to smart objects is given in [56], where the authors introduce and define Cognitive Objects for human-robot interaction and human-computer interaction and disambiguate them against existing 'Smart Objects'. Cognitive Objects incorporate self-awareness, reduce ambiguity and uncertainty in object recognition, and provide services to both humans and robots during their usage in real-world environments. In [57] the authors distinguish Cognitive Objects from other 'Smart Objects' and computationally enriched artifacts.

Since the time of the ISTAG scenarios, many years have passed and AmI becomes little by little a part of our everyday lives. Researchers keep on exploring the future and the next challenges, as according to [58] "there are strong indications that people have shifted their desires profoundly. This is partly due to the current financial crisis and partly due to the growing awareness that a sustainable development of our society calls for an approach different from the technology push of the past decades". This is what is called Synergetic Prosperity, which is the balance of People, Planet and Profit, which means that the term is "referring to meaningful digital solutions that balance mind and body, and 
community and earth thus contributing to a prosperous and sustainable development of mankind".

\section{Conclusion}

AmI seems to be here to stay. It is becoming a new trend with significant impact on the human-machine interaction and, as of this, to the design of technological solutions and consumer products. Although based on relatively solid foundations, such as the fields of sensors, electronics, networks (wired or wireless), machine learning and artificial intelligence, it is the very nature of the users that AmI systems have to be adapted to that extends the complexity of the adaptivity that the systems should exhibit. Subjects such as the inner system awareness and understanding, adaptivity to ever changing environments and legal issues are still open for further research and development.

\section{References}

[1] W. Weber, J. M. Rabaey, and E. Aarts, Eds., Ambient Intelligence, New York, NY: Springer-Verlag, 2005.

[2] E. Zelkha, B. Epstein, S. Birrell and C. Dodsworth, From Devices to "Ambient Intelligence", Digital Living Room Conference, June 1998.

[3] N. Dimitrova, Ambient Intelligence: A multimedia perspective, IEEE Computer Society, 2004.

[4] Gurevych, Max Mühlhäuser and Iryna. Introduction to Ubiquitous Computing. Chapter 1.1. s.l.: IGI Global, 2001.

[5] Online at http://www.oxygen.lcs.mit.edu, last accessed May 2011.

[6] Online at http://www.research.philips.com/cgibin/search.cgi?cc=1\&URL=http:\%2F\%2Fwww.research.philips.com\%2Ftec hnologies\%2Fprojects\%2Fhomelab\%2Findex.html\&q=icat\&wm=wrd, last accessed May 2011.

[7] IST Advisory Group, Scenarios for Ambient Intelligence. European Commission, 2001.

[8] IST Advisory Group, Strategic Orientations \& Priorities for IST in FP6. European Commission, 2002.

[9] IST Advisory Group, Ambient Intelligence: From Vision to Reality, European Commission, 2003.

[10] D. Wright, S. Gutwirth, M. Friedewald, E. Vildjiounaite, and Y. Punie, Y. (Eds.), Safeguards in a World of Ambient Intelligence, Series: The International Library of Ethics, Law and Technology, Vol. 1, 2010.

[11] Aarts, E., Harwig, R., Schuurmans, M., chapter "Ambient Intelligence" in The Invisible Future: The Seamless Integration Of Technology Into Everyday Life, McGraw-Hill Companies.

[12] Carlos Ramos, Juan Carlos Augusto, Daniel Shapiro. Ambient intelligence the next step for artificial intelligence. IEEE Computer Society. 2008. 
[13] Aliaksei Andrushevich, Marius Staub, Rolf Kistler, Alexander Klapproth. Towards Semantic Buildings: Goal-driven approach for building automation service allocation and control. 2010, 978-1-4244-6850-8/10.

[14] Kevin Bouchard, Amir Ajroud, Bruno Bouchard and Abdenour Bouzouane. SIMACT: A 3D Open Source Smart Home Simulator for Activity Recognition. T.H. Kim and H. Adeli (Eds.): AST/UCMA/ISA/ACN 2010, LNCS 6059, pp. 524-533. Springer-Verlag Berlin Heidelberg 2010, 2010.

[15] Jun-young Kwak, Pradeep Varakantham, Milind Tambe, Laura Klein, Farrokh Jazizadeh, Geoffrey Kavulya, Burcin B. Gerber, David J. Gerber. Towards Optimal Planning for Distributed Coordination Under Uncertainty in Energy Domains. Workshop on Agent Technologies for Energy Systems (ATES) at AAMAS 2011. 2011.

[16] Razvan Beuran, Junya Nakata, Yasuo Tan and Yoichi Shinoda. IEEE 802.15.4 Network Emulation Test bed. 2011 International Conference on Advanced Information Networking and Applications. DOI 10.1109/AINA.2011.25.

[17] Harshith. C, Karthik. R. Shastry, Manoj Ravindran, M.V.V.N.S Srikanth, Naveen Lakshmikhanth. Survey On Various Gesture Recognition Techniques For Interfacing Machines Based On Ambient Intelligence. International Journal of Computer Science \& Engineering Survey (IJCSES). 2010, Vol. Vol.1, No.2.

[18] Franz J. Kurfess and Greg Flanagan, Mehul Bhatt. Spatial Interactions between Humans and Assistive Agents. Help Me Help You: Bridging the Gaps in Human-Agent Collaboration, the AAAI 2011 Spring Symposium (SS-11-05). 2011.

[19] Heinzelman, Stanislava Soro andWendi. A Survey of Visual Sensor Networks. Advances in Multimedia. 2009, Article ID 640386.

[20] Zhangqin, He Jian, Dong Yumin, Zhang Yong and Huang. Creating an Ambient-Intelligence Environment Using Multi-Agent System. The 2008 International Conference on Embedded Software and Systems Symposia (ICESS2008). 2008.

[21] Ramos, Hoon Ko and Carlos. A Survey of context classification for intelligent systems research for Ambient Intelligence. 2010 International Conference on Complex, Intelligent and Software Intensive Systems. 2010.

[22] Zuraini Zainol, Keiichi Nakata,. Generic Context Ontology Modeling A Review and Framework. 2nd International Conference on Computer Technology and Development (ICCTD 2010). 2010.

[23] Kris McGlinn, Rob Brennan, Dave Lewis. The SimCon Generator: An Interactive Context Simulator for Rapid Evaluation of Smart Building Applications using Virtual Reality. 8th IEEE International Workshop on Managing Ubiquitous Communications and Services. 2011, 978-1-61284936-2/11. 
[24] Keith Worden, Wieslaw J. Staszewski and James J. Hensman. Natural computing for mechanical systems research: A tutorial overview. Mechanical Systems and Signal Processing. 2010.

[25] Chunchen Liu, Dayou Liu, Shengsheng Wang. Situation Modeling and Identifying under Uncertainty. 2010 Second Pacific-Asia Conference on Circuits, Communications and System (PACCS). 2010.

[26] Ainara Garzo, Iraitz Montalbán, Enrique León and Sarah Schlatter. Sentient: An approach to Ambient Assisted Emotional Regulation. International Symposium on Ambient Intelligence. 2010, Guimaraes, Portugal.

[27] Plexousakis, Theodore Patkos and Dimitris. EPIS-TEMIC Reasoning For Ambient Intelligence. Proceedings of the 3rd International Conference on Agents and Artificial Intelligence. 2011, DOI: 10.5220/0003149902420248.

[28] Patkos, T. and Plexousakis, D. (2009). Reasoning with Knowledge, Action and Time in Dynamic and Uncertain Domains. In IJCAI'09, pp. 885-890.

[29] Janneke Verhaegh, Willem Fontijn, Emile Aarts, Laurens Boer and Doortje van de Wouw. A development support bubble for children. Journal of Ambient Intelligence and Smart Environments. 2011, DOI 10.3233/AIS2011-0092.

[30] M. Ahad, J. K. Tan, H. Kim, and S. Ishikawa, Approaches for global based action representations for games and action understanding", in Proceedings of the International Workshop on Facial and Bodily Expressions for Control and Adaptation of Games (ECAG'11), Santa Barbara, CA, 2011, pp. 1-6.

[31] C. Shan, "An efficient approach to smile detection", in Proceedings of the International Workshop on Facial and Bodily Expressions for Control and Adaptation of Games (ECAG'11), Santa Barbara, CA, 2011, pp. 1-6.

[32] J. Shen, W. Shi, and M. Pantic, "HCI-2 workbench: A development tool for multimodal human-computer interaction systems", in Proceedings of the International Workshop on Facial and Bodily Expressions for Control and Adaptation of Games (ECAG'11), Santa Barbara, CA, 2011, pp. 1-8.

[33] J. Geigel and M. Schweppe, "Motion capture for realtime control of virtual actors in live, distributed, theatrical performances", in Proceedings of the International Workshop on Facial and Bodily Expressions for Control and Adaptation of Games (ECAG'11), Santa Barbara, CA, 2011, pp. 1-6.

[34] Carlos Antonio Acosta Calderon, Rajesh Elara Mohan, Changjiu Zhou. Elderly Telecare, in the Singapore Context. HRI 2011 Workshop. 2011.

[35] Annica Kristoffersson, Silvia Coradeschi, Amy Loutfi, Kerstin Severinson Eklundh. Towards Evaluation of Social Robotic Telepresence based on Measures of Social and Spatial Presence. 1st Workshop on Social Robotic Telepresence held at HRI 2011. 2011.

[36] Constantinescu, Monica Vladoiu and Zoran. U-learning within a contextaware multiagent environment. International Journal of Computer Networks \& Communications (IJCNC). 2011, Vol. Vol.3, No.1.

[37] Boris Mejias, Alfredo Cadiz, Peter Van Roy, Kim Mens. A Self-Adaptable Network Topology for Ambient Intelligence. Second IEEE International 
Conference on Self-Adaptive and Self-Organizing Systems Workshops. 2008.

[38] Parisa Rashidi, Diane J. Cook. Activity knowledge transfer in smart environments. Pervasive and Mobile Computing. 2011, doi:10.1016/j.pmcj.2011.02.007.

[39] Paradells, Carles Gomez and Josep. Wireless Home Automation Networks: A Survey of Architectures and Technologies. IEEE Communications Magazine. 2010.

[40] Nakajima, Hiroaki Kimura and Tatsuo. Extracting Key Factors to Design Applications in Ambient Intelligence Environment. IEEE/WIC/ACM International Conference on Web Intelligence and Intelligent Agent Technology. 2008.

[41] Hui-Huang Hsu, Po-Kai Chen, and Chi-Vi Lin. RFID-Based Danger Prevention for Home Safety, 2010 2nd International Symposium on Aware Computing (ISAC), 1-4 Nov. 2010.

[42] Hoon Ko, Jongmyung Choi, Maricel O. Balitanas, Taihoon Kim and Carlos Ramos. A Study on Secure Contents Using in Intelligent Urban Computing. International Journal of Smart Home. 2010, Vol. Vol.4, No.2.

[43] Dale DZEMYDIENE, Antanas Andrius BIELSKIS, Arunas ANDZIULIS, Darius DRUNGILAS, Gediminas GRICIUS. Recognition of Human Emotions in Reasoning Algorithms of Wheelchair Type Robots. INFORMATICA. 2010, Vol. Vol. 21, No. 4.

[44] Betke, Christopher Kwan and Margrit. Camera Canvas: Image Editing Software for People with Disabilities. Boston University Computer Science Technical. 2011, Vols. Report No. BUCS-TR-2011-007.

[45] Pavan Dadlani, Panos Markopoulos, Alexander Sinitsyn, Emile Aarts. Supporting peace of mind and independent living with the Aurama awareness system. Journal of Ambient Intelligence and Smart Environments. 2011, DOI 10.3233/AIS-2011-0094.

[46] Christoph Stahl, Jochen Frey, Jan Alexandersson and Boris Brandherm. Synchronized Realities. Journal of Ambient Intelligence and Smart Environments. 2011, DOI 10.3233/AIS-2011-0093.

[47] Carlos Lamsfus, Christoph Grün, Aurkene Alzua-Sorzabal and Hannes Werthner. Context-based semantic matchmaking to enhance tourists' experiences. Journal for the Informatics Professional (eingeladen). 2010, Vols. 203(2010), S. 17 - 23.

[48] Charles Callaway, Oliviero Stock, Elyon Dekoven, Kinneret Noy, Yael Citron, Yael Dobrin. Mobile Drama in an Instrumented Museum: Inducing Group Conversation via Coordinated Narratives. ACM 2011 International Conference on Intelligent User Interfaces Palo Alto, CA. 2011.

[49] Ning Tan, Gaëtan Pruvost, Matthieu Courgeon, Céline Clavel, Yacine Bellik, Jean-Claude Martin. A Location-Aware Virtual Character in a Smart Room: Effects on Performance, Presence and Adaptivity. IUI 2011, Palo Alto, California, USA. 2011, 978-1-4503-0419-1/11/02. 
[50] Terano, Hamed Khandan and Takao. Knowledge Request-Broker Architecture: A Platform for Social Computational Intelligence. International Journal of Energy, Information and Communications. 2010, Vol. Vol. 1, Issue. 1.

[51] Julian Schütte, Tobias Wahl. A Description Logic based Approach on Handling Inter-Domain Policy Conflicts using Meta-Policies. IEEE Vehicular Technology Magazine. 2010, Vol. Volume 5, Number 3.

[52] F. André, Daubert Erwan, Nain Grégory, M. Brice, and B. Olivier, "F4Plan: An Approach to build Efficient Adaptation Plans," in 7th International ICST Conference on Mobile and Ubiquitous Systems (Mobiquitous), Sydney, Australia, December 2010.

[53] Ioannis Panagiotopoulos, Lambrini Seremeti, Achilles Kameas, Vasilios Zorkadis. PROACT: An ontology-based model of privacy policies in Ambient Intelligence Environments. 2010, 14th Panhellenic Conference on Informatics. 2010.

[54] T. Heinrotha, A. Kameas, G. Pruvostc, L. Seremetib, Y. Bellikc and W. Minker. Human computer interaction in next generation ambient intelligence environments . Intelligent Decision Technologies 5 . 2011.

[55] Kameas, Achilles. Towards the Next Generation of Ambient Intelligent Environments. 2010 Workshops on Enabling Technologies: Infrastructure for Collaborative Enterprises. 2010, DOI 10.1109/WETICE.2010.58.

[56] Andreas Möller, Luis Roalter, Matthias Kranz. Cognitive Objects for Human-Computer Interaction and Human-Robot Interaction. HRI'11, Lausanne, Switzerland. 2011, ACM 978-1-4503-0561-7/11/03.

[57] Kameas, Achilles. Towards the Next Generation of Ambient Intelligent Environments. 2010 Workshops on Enabling Technologies: Infrastructure for Collaborative Enterprises. 2010, DOI 10.1109/WETICE.2010.58.

[58] Grotenhuis, Emile Aarts and Frits. Ambient Intelligence 2.0: Towards Synergetic Prosperity. Journal of Ambient Intelligence and Smart Environments. 2011, DOI 10.3233/AIS-2011-0090. 